\title{
The extreme physical conditions in the center of Mrk 876 and the switch on of its AGN activity
}

\section{Eugenio Bottacini*}

W. W. Hansen Experimental Physics Laboratory and Kavli Institute for Particle Astrophysics and Cosmology, Stanford University, USA

E-mail: eugenio.bottacini@stanford.edu

\section{Elena Orlando, Giacomo Vianello, Igor Moskalenko}

W. W. Hansen Experimental Physics Laboratory and Kavli Institute for Particle Astrophysics and Cosmology, Stanford University, USA

The trigger mechanism of Active Galactic Nucleus (AGN) activity has deep implications with the spin of the supermassive black hole (SMBH). However, the spin measurement is not straightforward. In this talk we present the results of a case study on Mrk 876. The detection of a transient and extreme gravitationally redshifted Fe-line allows us to find its emission mechanism, thereby constraining the rotation of the super-massive black hole in the center of Mrk 876. We also present predictions through simulations for the NUSTAR mission. The spin measurement through emission lines is one of the scientific topics of the future Athena mission.

The 34th International Cosmic Ray Conference,

30 July- 6 August, 2015

The Hague, The Netherlands

\footnotetext{
* Speaker.
} 


\section{Introduction}

The cosmic X-ray background $(\mathrm{CXB})$ is the result of the integrated emission from active galactic nuclei (AGNs). At their centers the photons from the accretion disk surrounding the supermassive black hole (SMBH) are inverse-Comptonized by electrons (hot corona) producing the primary radiation. It has become clear now that the correlation between bulge component and SMBH is tightly related to the spin of the SMBH [1]. Recent advances in detailed theoretical studies related to the spin of SMBHs by [2] predict that rapidly spinning black holes could be a major contributor to the yet unresolved peak of the CXB at $\sim 30 \mathrm{keV}$. Therefore, the detection of rotating black holes is important. A powerful tool for their detection are $\mathrm{X}$-ray spectral lines. Indeed, the primary radiation also irradiates the accretion disk that reflects it through several reprocessing steps [e.g. 3] including fluorescence emission of $\mathrm{K} \alpha$ lines of most abundant elements [4]. Therefore, emission lines from reflection spectra are good diagnostics of the environment of the inner region of AGN [5]. Among emission lines from abundant elements in AGN spectra, the most common is the $\mathrm{Fe} \mathrm{K} \alpha$ line at $6.4 \mathrm{keV}$.

Even more intriguing are transient fluorescence-emission lines. In this case the primary radiation can originate in magnetic field reconnection [7] dissipating the energy input into the Comptonizing corona and it irradiates a limited portion (hotspot) of the accretion disk for a short time. As a result the emerging fluorescence lines are transient. They are also affected by Doppler and gravitational effects causing distorted line shapes and photon energy shifts [6]. However, shifted line energies can be caused also by AGN outflows and inflows. These shifts to unexpected energies led to conflicting interpretations in the literature [8] that are comprehensively discussed in [9].

We present the results on a detection in a Swift/XRT observation of an emission line at $4.8 \mathrm{keV}$ rest-frame energy of the Seyfert type 1 AGN Mrk 876 studied in detail in [10].

\section{Line feature in Swift/XRT spectrum of Mrk 876}

Mrk 876 is part of an optically selected sample of the Palomar-Green (PG) Bright Quasar Survey sample [11]. The redshift of the host galaxy is $z=0.1385$ [12] corresponding to 551.4 Mpc for $H_{0}=73 \mathrm{~km} \mathrm{~s}^{-1} \mathrm{Mpc}^{-1}$ assuming Hubble flow. The Galactic absorption toward Mrk 876, assuming solar abundance, has been measured by [13] using NRAO $140 \mathrm{ft}$ telescope of Green Bank finding a value of $\mathrm{N}_{H}^{\text {gal }}=2.66 \times 10^{20}$ atoms $\mathrm{cm}^{-2}$ with $5 \%$ error. The source was observed by Swift/XRT (obs id: 00050300004) on 2005-03-01. For the analyses of the observation we used HEAsoft 6.11.1. Events for the spectral analysis are extracted from a circular region of interest centered on the source position having a radius of $\sim 20$ pixels (corresponding to $\sim 47 \operatorname{arcsec}$ ). The background is extracted from a nearby source-free circular region of interest of 50 pixel radius. The best fit model for the background-subtracted source spectrum is obtained by adding to the power-law a Gaussian-component at $4.22 \mathrm{keV}$ that models well the residuals (see Figure 1). All the parameters of the model are free to float obtaining a line energy of $4.22_{-0.04}^{+0.05} \mathrm{keV}$ and a width of $90_{-75}^{+76} \mathrm{eV}$ compatible with the energy resolution of $\sim 100 \mathrm{eV}$. 


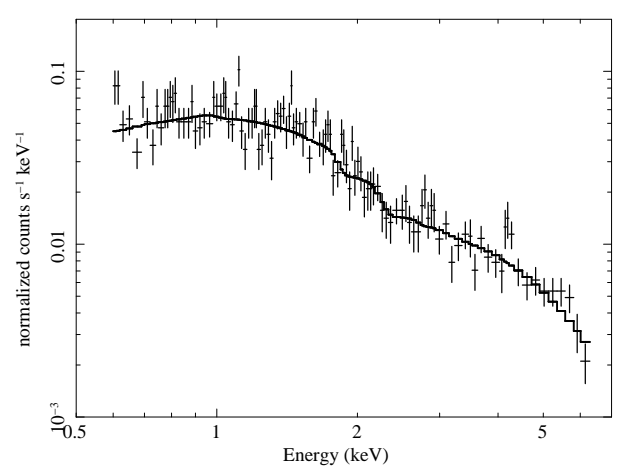

Figure 1: Swift/XRT spectrum of Mrk 876 (observer's frame). Excess at $4.22 \mathrm{keV}$.

\subsection{Statistical significance of the line feature at $4.22 \mathrm{keV}$ observed-frame energy}

There is general consensus that line searches justified by observational data must be in need of validation through randomized trials [14]. [9] used such an approach that we adopt with further improvements.

To estimate the significance of the line at $4.22 \mathrm{keV}$ observed-frame energy, we carry out Monte Carlo simulations in the wide energy range of $0.6-6.5 \mathrm{keV}$. For the fitting procedure we have used the $\chi^{2}$ minimization applying Churazov-weighting [15] and $C$-statistic [16] based on maximumlikelihood ratio method. A detailed description of this approach can be found in [10].

As a result, we find that the measured spectrum being described by a power law (without the gaussian feature) is rejected with a probability of roughly $\sim 99 \%$. The tests for systematic effects in the spectrum are discussed in [10].

\subsection{Further X-ray observations}

Mrk 876 was targeted by Swift/XRT with further 16 observations. We have reanalyzed also the GINGA [17] spectrum of Mrk 876 as published in [18] and XMM-Newton observations [19] and [23]. Spectra were analyzed with XSPEC 12 [21]. A table with all analyzed observations can be found in [10].

\section{Discussions}

The importance of X-ray spectral lines at unforeseen energies is their capability to shed light on the extreme physical conditions where they originate. Any new detection is important in light also of future missions with improved throughput and energy resolution [e.g. Athena $+; 22]$.

\subsection{The line feature in Mrk 876}

Being a Seyfert type 1 AGN, Mrk 876 allows for clean view on the innermost accretion region. As such, it is an ideal target for a case study. None of the GINGA, Swift/XRT, and XMM-Newton observations reveal the narrow $\mathrm{Fe} \mathrm{K} \alpha$ line at $6.4 \mathrm{keV}$. This is in agreement with previous analyses of GINGA observation [18] and XMM-Newton observations [23; 19]. This line, often seen in AGN, is interpreted as Fe fluorescence from cold (neutral) matter far from the inner accretion disk [24; 25]. A viable explanation for its absence in Mrk 876 can come from the X-ray Baldwin effect [26], an 
anti-correlation between the equivalent width of the Fe $\mathrm{K}$ line and $\mathrm{X}$-ray luminosity, which can be explained by the drop of the covering factor of the molecular AGN torus [25]. Therefore, it is not surprising that Mrk 876 does not display a steady $\mathrm{Fe} \mathrm{K} \alpha$ line being one of the most luminous AGN among the systematically studied Palomar-Green (PG) Bright Quasar Survey sample at X-rays [23]. However, for one Swift/XRT observation (obs id: 00050300004) we find a significant line emission at a confidence level of $99 \%$ at $4.80_{-0.04}^{+0.05} \mathrm{keV}$ rest-frame energy. At the same rest-frame energy a similar line but with lower confidence level has been reported by [27] for Mrk 841 that is a Seyfert type 1 AGN.

\subsection{On the origin of the $4.8 \mathrm{keV}$ rest-frame line}

In the following we consider the possible origin of our transient line in the accretion disk hotspot scenario [28; 29]. The inclination angle of Mrk 876's accretion disk of $15.4_{-6.8}^{+12.1} \mathrm{deg}$ is independently constrained by [30]. This very low inclination angle leads to the gravitational redshift dominance of the line over the Doppler effect in both rotating (Kerr) black hole [31] and a non-rotating (Schwarzschild) black hole environments [32]. As a result the line profile tends to a single peak [32], which is intrinsically very narrow $[<100 \mathrm{eV} ; 28]$ and mainly subject to gravitational redshift. The observed gravitational redshift factor is $v_{\text {observed }} / v_{\text {rest frame }}=0.75_{-0.01}^{+0.01}$ where errors account for the line energy uncertainty. Following [32] this factor cannot be obtained by a non-rotating black hole. We constrain the emission-region on the accretion disk by simulating the model of [31] accounting for its different emissivity power-law indices and taking care of the uncertainties of the inclination angle of the source. The gravitational redshift factor of $0.75_{-0.01}^{+0.01}$ cannot be obtained from emission-regions at radii above $6 r_{g}$ (where $r_{g}=G M_{B H} / c^{2}$ ) reaching at most a factor of 0.89 . On the contrary for radii below $6 r_{g}$ such a gravitational redshift factor can be well reproduced. Only accretion disks of Kerr black holes can extend below $6 r_{g}$ [34] emphasizing once again the environment of a rotating black hole. On the other hand, for Schwarzschild black holes, a detailed line profile study by [35] allows for the fluorescence $\mathrm{Fe} \mathrm{K} \alpha$ line emission from material spiraling toward the event horizon even below $6 r_{g}$. However, the profile is predicted to be doublepeaked for accretion disk inclination angles other than face-on, in contrast to our finding. As a result, the line can be reproduced by a rotating black hole only. This is in agreement with a range of spectral parameters predicted in an accurate study by [36] for a black hole with angular momentum $a=0.9$ and disk inclination $\theta_{0}=30$ deg for a hotspot on an accretion disk emitting photons from its last stable orbit $r_{m s}+d r$. Unfortunately the low signal-to-noise spectrum does not allow for a more constraining fit with the Laor-model or with the more complex kerrdisk-model. The same low signal-to-noise ratio prevents from attempting a cross-correlation study between the line light curve and the continuum light curve that are predicted to correlate in the hotspot framework [36]. Also correlation studies between Swift/XRT and Swift/BAT are impossible due to the low signal-to-noise observations of the latter [33]. Unfortunately, no UVOT data were taken, which could constrain the geometry of the emitting region [4]. On the other hand the independent estimates of the black hole mass of Mrk $876[37 ; 30]$ allow us to compute the orbital period for the hotspot on the accretion disk. [36] predict the line to appear for a relatively short fraction of the total orbital period of the hotspot at the distance from the black hole where the line occurs. Thus, we estimate the orbital period using the formula below of [38] as performed in [36] where $r$ is in 
units of gravitational radii.

$$
P=310\left(r^{\frac{3}{2}}+a\right) \frac{M_{B H}}{10^{7} M_{\odot}} \quad[\mathrm{s}] .
$$

We assume the photons emitted from a spinning black hole $a=0.9$ at a disk radius of $6 r_{g}$. Taking the two independently inferred black hole masses of Mrk $876 M_{B H}=1.3 \times 10^{9} \mathrm{M}_{\odot}$ [30] and $M_{B H}=2.4 \times 10^{8} \mathrm{M}_{\odot}$ [37], the resulting orbital periods are $\mathrm{P}=628 \mathrm{ksec}$ and $\mathrm{P}=135 \mathrm{ksec}$, respectively. Both are significantly longer than the Swift/XRT observation time $(\sim 25 \mathrm{ksec})$. This is in agreement with the narrow lines to appear for a fraction of the total period as the flare dies out or emission gets below detectability [36]. The Swift/XRT observation (obs id: 00050302001) taken 6 days later without any line detection, makes the hotspot scenario a consistent picture. Also the line feature has never been observed in any other X-ray observation confirming the transient nature of the event that reinforces the hotspot origin.

Another hypothetical origin of the line could be the emission from enhanced abundances of sub-Fe elements resulting from Fe spallation. In this scenario, Fe nuclei hit by cosmic-rays are converted into elements of atomic number $Z$ below Fe. The element emitting closest to our line energy would be Vanadium at $4.9 \mathrm{keV}$ (rest-frame energy). However, the absence of the $\mathrm{Fe} \mathrm{K} \alpha$ at $6.4 \mathrm{keV}$, which would still be the strongest line in this scenario [39], makes it an unlikely explanation. Also the transient nature of the line would be difficult to explain.

In a further hypothesis a $\sim 4.7 \mathrm{keV}$ (rest-frame energy) spectral feature could originate also from Compton down-scattered Fe line from FeXXV at $6.7 \mathrm{keV}$ [40]. However, the resulting gaussian component is expected to be much broader $(\sim 0.4 \mathrm{keV})$. Also, this component needed to be present in all our analyzed Mrk 876 spectra. Thus, the down-scattered Fe line is also very unlikely.

A redshifted $\mathrm{Fe}$ line could originate also from inflows and outflows of material (not related to the accretion disk) in the vicinity of the black hole. In this region the material would be subject to strong gravitational effects reaching mildly relativistic $(\sim 0.4 \mathrm{c})$ velocities [41] causing an energy shift of emission lines. This is the interpretation for a line shift observed in Mrk 766 in a detailed and time-resolved study by [42] in presence of a strong Fe $\mathrm{K} \alpha$ component at $6.4 \mathrm{keV}$. In fact, the absence of this latter component in all our analyzed observations of Mrk 876 disfavors this interpretation since it is difficult to imagine one single blob of material emitting an Fe line. In addition our observations a month earlier (obs id: 00050300005) and 6 days later (obs id: 00050302001) with Swift/XRT should have detected the corresponding $6.4 \mathrm{keV}$ line.

\subsection{On the significance of the line}

A detailed study by [8] suggests that reported line significances might be altered by a publication bias where only most significant among detected spectral features are mentioned. Such an important issue can be addressed by a statistical study of a sample as a whole where several lower significance lines are detected as e.g. in [41]. Instead, our study reports a case study of a transient event in the hotspot scenario. Therefore, to determine the detection likelihood we cannot incorporate the non-presence of the lines in other observations. Also is it here impossible to address the publication bias as it must address the bias for a source population at large [8].

\subsection{Predictions of spectral features at energies above $15 \mathrm{keV}$}

The radiative efficiency of the accretion process is enhanced in rotating SMBHs because the 
accretion disk reaches close to the black hole. This increased efficiency has been studied in detail in a very recent work by [2] predicting that a large fraction of the CXB at energies $\sim 30 \mathrm{keV}$ could be due to rotating SMBHs. Therefore we simulated the expected spectrum of Mrk 876 at hard $\mathrm{X}$-ray energies accounting for the reflection hump predicted to peak in this energy range [5]. The spectrum is simulated with XSPEC using NUSTAR response files. Source extraction is simulated using background data for a 30 arcsec (bkgd_300arsec.pha) region and an off-axis distance of 1 arcmin (point_30arcsecRad_1arcminOA.arf). Since available Swift/XRT data show stable spectral data and fluxes, we fit these spectra simultaneously to the recently improved reflection model RELXILL [43] that is a self-consistent physical model. The result is shown in Figure 2. The derived flux in the $3-6 \mathrm{keV}$ band is used to carefully evaluate the NuSTAR exposure by matching the same flux in the same energy band and using the same RELXILL model with the same parameters. Swift/XRT data and NUSTAR data can be directly compared as they display the real power output (Y-axis $E^{2} d N / d E$. The dimensionless black hole spin $a$ is 0.9 . The comparison between the power-law fit (light red dots) to XRT data only and the simulated NuSTAR data (red crosses) shows that the hump can be clearly seen.

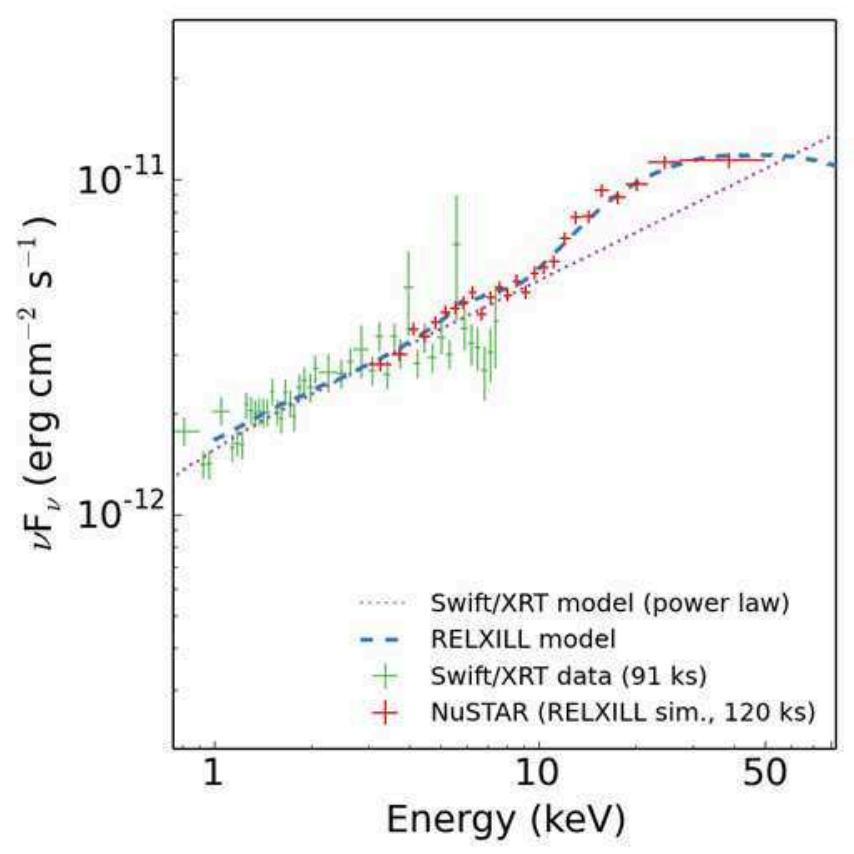

Figure 2: Swift/XRT spectra (green) fitted simultaneously with RELXILL model (power law). NuSTAR simulated data (red).

\section{Conclusions}

Our analysis of the Swift/XRT observation of Mrk 876 (obs id: 00050300004) has revealed an emission line at $4.80_{-0.04}^{+0.05} \mathrm{keV}$ rest-frame energy associated to the source. The line is detected at $\sim 99 \%$ confidence level through Monte Carlo simulations using $\chi^{2}$ and $C$-statistic. The possible origin of the line is the hotspot scenario in which a flare in the hot corona (possibly due to 
magnetic reconnection) illuminates a limited portion of the accretion disk that emits the Fe line by fluorescence. Due to the independent constraints of the low inclination of Mrk 876's accretion disk (15.4 deg) and the observed gravitational redshift, we can constrain that the line is emitted from the accretion disk at radii below $6 r_{g}$ (where $r_{g}=G M_{B H} / c^{2}$ ) from a Kerr black hole. The alternative possible origins due to Fe spallation, down-scattered Fe line, and mass inflows/outflows are disfavored in light of the absence of the $6.4 \mathrm{keV}$ Fe line and the variability studies due to the many available observations of Swift/XRT, XMM-Newton, and GINGA.

To the best of our knowledge, this is the first time that a transient line revealed at $99 \%$ confidence level is unambiguously associated to the hotspot scenario.

E.B. acknowledges NASA grant NNX13AF13G.

\section{References}

[1] Volonteri, M., Sikora, M., Lasota, J.-P., \& Merloni, A. 2013, ApJ, 775, 94

[2] Vasudevan, R. V., Fabian, A. C., Reynolds, C. S., \& Gallo, L. C. 2015, arXiv:1506.01027

[3] Matt, G., Perola, G. C., \& Piro, L. 1991, A\&A, 247, 25

[4] George, I. M., \& Fabian, A. C. 1991, MNRAS, 249, 352

[5] Ross, R. R., \& Fabian, A. C. 1993, MNRAS, 261, 74

[6] Fabian, A. C., Iwasawa, K., Reynolds, C. S., \& Young, A. J. 2000, PASP, 112, 1145

[7] Merloni, A., \& Fabian, A. C. 2001, MNRAS, 328, 958

[8] Vaughan, S., \& Uttley, P. 2008, MNRAS, 390, 421

[9] Turner, T. J., Miller, L., Reeves, J. N., Lobban, A., Braito, V., Kraemer, S. B., \& Crenshaw, D. M. 2010, ApJ, 712, 209

[10] Bottacini, E., Orlando, E., Greiner, J., et al. 2015, ApJ, 798, L14

[11] Schmidt, M., \& Green, R. F. 1983, ApJ, 269, 352

[12] Lavaux, G., \& Hudson, M. J. 2011, MNRAS, 416, 2840

[13] Elvis, M., Wilkes, B. J., \& Lockman, F. J. 1989, AJ, 97, 777

[14] Protassov, R., van Dyk, D. A., Connors, A., Kashyap, V. L., \& Siemiginowska, A. 2002, ApJ, 571,545

[15] Churazov, E., Gilfanov, M., Forman, W., \& Jones, C. 1996, ApJ, 471, 673

[16] Cash, W. 1979, ApJ, 228, 939

[17] Makino, F. 1987, Astrophys. Lett., 25, 223 
[18] Lawson, A. J., \& Turner, M. J. L. 1997, MNRAS, 288, 920

[19] Piconcelli, E., Jimenez-Bailón, E., Guainazzi, M., Schartel, N., Rodríguez-Pascual, P. M., \& Santos-Lleó, M. 2005, A\&A, 432, 15

[20] Porquet, D., Reeves, J. N., Uttley, P., \& Turner, T. J. 2004, A\&A, 427, 101

[21] Arnaud, K. A. 1996, in Astronomical Society of the Pacific Conference Series, Vol. 101, Astronomical Data Analysis Software and Systems V, ed. G. H. Jacoby \& J. Barnes, 17

[22] Nandra, K., Barret, D., Barcons, X., et al. 2013, arXiv:1306.2307

[23] Porquet, D., Reeves, J. N., O’Brien, P., \& Brinkmann, W. 2004, A\&A, 422, 85

[24] Yaqoob, T., \& Padmanabhan, U. 2004, ApJ, 604, 63

[25] Page, K. L., O’Brien, P. T., Reeves, J. N., \& Turner, M. J. L. 2004, MNRAS, 347, 316

[26] Iwasawa, K., \& Taniguchi, Y. 1993, ApJ, 413, L15

[27] Petrucci, P. O. et al. 2007, A\&A, 470, 889

[28] Nayakshin, S., \& Kazanas, D. 2001, ApJ, 553, L141

[29] Turner, T. J. et al. 2002, ApJ, 574, L123

[30] Bian, W., \& Zhao, Y. 2002, A\&A, 395, 465

[31] Laor, A. 1991, ApJ, 376, 90

[32] Fabian, A. C., Rees, M. J., Stella, L., \& White, N. E. 1989, MNRAS, 238, 729

[33] Baumgartner, W. H., Tueller, J., Markwardt, C. B., Skinner, G. K., Barthelmy, S., Mushotzky, R. F., Evans, P. A., \& Gehrels, N. 2013, ApJS, 207, 19

[34] Thorne, K. S. 1974, ApJ, 191, 507

[35] Reynolds, C. S., \& Begelman, M. C. 1997, ApJ, 488, 109

[36] Dovčiak, M., Bianchi, S., Guainazzi, M., Karas, V., \& Matt, G. 2004, MNRAS, 350, 745

[37] Kaspi, S., Smith, P. S., Netzer, H., Maoz, D., Jannuzi, B. T., \& Giveon, U. 2000, ApJ, 533, 631

[38] Bardeen, J. M., Press, W. H., \& Teukolsky, S. A. 1972, ApJ, 178, 347

[39] Skibo, J. G. 1997, ApJ, 478, 522

[40] Fabian, A. C. et al. 2012, MNRAS, 419, 116

[41] Tombesi, F., Cappi, M., Reeves, J. N., Palumbo, G. G. C., Yaqoob, T., Braito, V., \& Dadina, M. 2010, A\&A, 521, A57

[42] Turner, T. J., Kraemer, S. B., \& Reeves, J. N. 2004, ApJ, 603, 62

[43] Dauser, T., García, J., Parker, M. L., Fabian, A. C., \& Wilms, J. 2014, MNRAS, 444, L100 